\title{
HYVET-Studie
}

\section{Blutdrucksenkung auch im hohen Alter lohnenswert}

\section{Eine konsequente antihypertensive Therapie mit der Fixkombination Indapamid plus Perindopril schützt umfassend vor den Folgen eines Blut- hochdrucks. Typ-2-Diabetiker und hochbetagte Patienten profitieren besonders von den protektiven Effekten der Kombinationstherapie.}

Die European Society of Hypertension (ESH) empfiehlt in ihrer kürzlich überarbeiteten Leitlinie generell einen Blutdruckzielwert von $140 / 90 \mathrm{mmHg}$. Die stringenten Zielwerte wurden teilweise etwas gelockert, so Prof. Jürgen Scholze, Berlin. Bei über 80-Jährigen empfiehlt die ESH nun Blutdruckzielwerte von unter 150/80 $\mathrm{mmHg}$. Das lasse sich am besten mit einer initialen Kombinationstherapie realisieren. Grundlage für diese Empfehlung sind die Daten der HYVET-Studie, die erstmals die Auswirkungen einer Blutdrucksenkung gezielt bei über 80 -jährigen Hypertonikern untersucht hat. Unter einer Kombinationstherapie mit Perindopril/Indapamid kam es zu einer signifikanten Reduktion der
Gesamtmortalität (-21\%), der Schlaganfallrate $(-30 \%)$ und der Herzinsuffizienzrate (-64\%) gegenüber Placebo. „Die Patienten, die in Ihre Praxis kommen, sind nicht zu alt für eine sinnvolle Hochdrucktherapie“, konstatierte Scholze.

Prof. Christian Sieber, Nürnberg, wies darauf hin, dass eine Hypertonie nur selten allein kommt. Gerade im Alter sei ein Bluthochdruck häufig kombiniert mit KHK, Niereninsuffizienz und insbesondere mit Typ-2-Diabetes. Hier sei Perindopril//ndapamid (Preterax ${ }^{\circledR} \mathrm{N}$, BiPreterax ${ }^{\circledR} \mathrm{N}$ ) eine sinnvolle Therapieoption.

Die ADVANCE-Studie ergab, dass Diabetiker durch diese Kombination vor den makro- und mikrovaskulären Komplikationen ihrer Erkrankung geschützt werden. Die kardiovaskuläre Mortalität sank um $18 \%$, das Risiko für koronare Ereignisse um $14 \%$ und für renale Ereignisse um $21 \%$, jeweils signifikant gegenüber Placebo. Das relative Risiko für die Entwicklung oder Verschlechterung einer Albuminurie konnte im Vergleich zu Placebo um 22\% verringert werden. Gleichzeitig stieg die Häufig- keit einer Regression der Eiweißausscheidung signifikant um $16 \%$. Die Effekte waren unabhängig vom Ausgangsblutdruck und von einer antihypertensiven Begleitmedikation.

(aam) II

\section{Vorsymposium des AK Hypertonie im BDI e.V., 116 Kongress der DGIM, Wiesbaden, 10. April 2010 (unterstützt von Servier)}

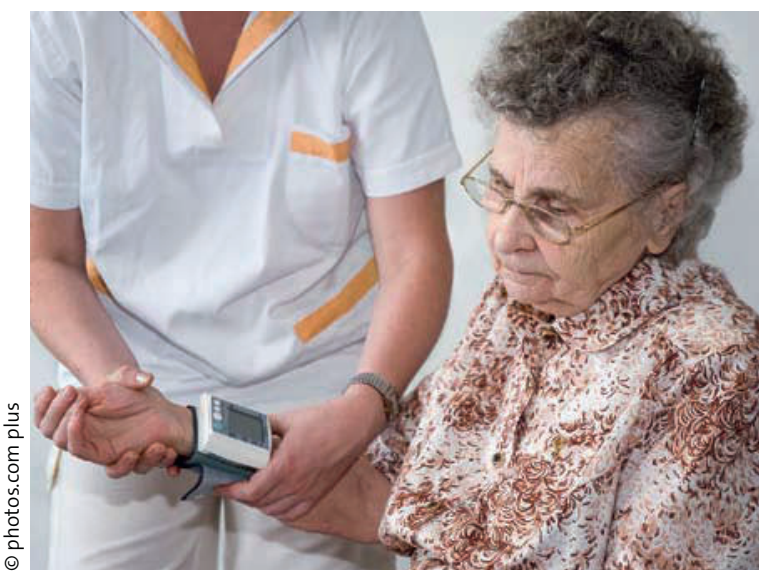

Auch Senioren profitieren von einer antihypertensiven Therapie.

\section{Pulmonale Hypertonie}

\section{PDE-5-Hemmer kann Zeit bis zur Transplantation überbrücken}

Der Phosphodiesterase-5-Inhibitor Sildenafil kann bei Patienten mit einer sekundären pulmonalen Hypertonie den pulmonalen Gefäßwiderstand senken und könnte für sie eine sinnvolle Therapieoption sein, berichtete Dr. Uwe Schulz aus Bad Oeynhausen.

Bei 30-40\% der Patienten mit terminaler Herzinsuffizienz entwickelt sich als Konsequenz einer diastolischen Dysfunktion eine sekundäre pulmonale Hypertonie $(\mathrm{PH})$. Eine mangelhafte rechtsventrikuläre Funktion verschlechtert die Prognose dieser Patienten zusätzlich. Als einzige Option bleibt oft eine Herztransplantation.

Sildenafil $\left(\right.$ Revatio $\left.^{\circledR}\right)$ ist für diese Patienten derzeit nur als Off-label-Option ver- fügbar. Daten zur sublingualen Therapie mit Sildenafil zeigen, dass nach 30-45 Minuten akut der pulmonalarterielle Druck abgesenkt und der Lungenwiderstand und der transpulmonale Gradient verbessert werden. Auch eine vorübergehende, etwa eine Stunde anhaltende Absenkung des Wedge-Drucks sowie die transiente Verbesserung des Herzzeitvolumens nach der Einmalgabe sprechen dafür, dass Sildenafil eine sinnvolle Option bei diesen Patienten sein könnte, so Schulz.

In einer kleinen placebokontrollierten Studie war unter Sildenafil die Hospitalisierungsrate reduziert. Für die Therapie über die Transplantation hinaus existieren Daten aus einer kleinen Fallserie, wonach ein vorübergehender transplantationsbe- dingter Anstieg des Herzzeitvolumens, des pulmonalvaskulären Widerstands und des transpulmonalen Gradienten effektiv behandelt wird.

Schulz machte mit dieser Strategie bei 29 Patienten, zumeist mit KHK oder dilatativer Kardiomyopathie, die Erfahrung, dass bei einer mittleren Behandlungsdauer mit Sildenafil von 300 Tagen eine tolerable Absenkung des mittleren Perfusionsdrucks, ein Absinken des rechtsventrikulären Drucks und eine Verbesserung des Cardiac Index sowie der systemischen und pulmonalen Widerstände eintreten.

(hae) II

Symposium beim Kongress der DGK, Mannheim, April 2010 (Veranstalter: Pfizer) 